Résumés des conférences et travaux

\title{
Histoire de Paris
}

\section{Guy-Michel Leproux}

\section{OpenEdition \\ Journals}

Édition électronique

URL : https://journals.openedition.org/ashp/1025

DOI : 10.4000/ashp.1025

ISSN : 1969-6310

Éditeur

Publications de l'École Pratique des Hautes Études

Édition imprimée

Date de publication : 2 février 2011

Pagination : 246-249

ISSN : 0766-0677

\section{Référence électronique}

Guy-Michel Leproux, « Histoire de Paris », Annuaire de l'École pratique des hautes études (EPHE), Section des sciences historiques et philologiques [En ligne], 141 | 2011, mis en ligne le 24 février 2011, consulté le 06 juillet 2021. URL : http://journals.openedition.org/ashp/1025 ; DOI : https://doi.org/10.4000/ ashp. 1025 


\title{
HISTOIRE DE PARIS
}

\author{
Directeur d'études : M. Guy-Michel LeProux
}

Programme de l'année 2008-2009 : I. Recherches sur les artistes parisiens, XV'-XVII ${ }^{e}$ siècles. - II. Le théâtre à Paris au XVI siècle.

\section{Recherches sur les artistes parisiens, $X V^{e}-X V I I^{e}$ siècles}

Plusieurs questions ont été abordées au cours de l'année. On s'est d'abord interrogé sur les sculpteurs parisiens qui ont pu travailler sous la direction de Philibert Delorme au château d'Anet. On savait depuis les travaux de Maurice Roy que Jean Mayart, sculpteur à Paris, avait été employé en 1555 à reparer en cire le groupe constitué d'un cerf et de quatre chiens destiné au couronnement du portail, l'ensemble devant être fondu en bronze par Benoît Leboucher. Le nom de ce Mayart n'apparaît pas dans les comptes royaux. Roy avait seulement pu constater qu'il était voisin de Philibert Delorme rue de la Cerisaie et qu'un Antoine Mayart, tailleur d'images, qui lui était sans doute apparenté, figurait sur un registre de baptêmes d'Avon aux côtés de la fille de l'architecte. En fait, l'artiste est bien cité dans des sources d'archives, mais sous un autre nom : un acte notarié mentionne en effet en 1559 le remariage de Jeanne Delante, veuve en secondes noces de feu Jehan Mayart, dict Crestien, en son vivant sculpteur. Il n'y a guère de doute sur l'identité de l'artiste, puisque Antoine Mayart, frère du défunt, intervient dans le contrat. Or, Jean Chrétien est, lui, bien connu : il fut notamment employé par Pierre Lescot au décor des voûtes de l'une des volées du grand escalier du Louvre, et il est répertorié dans les comptes de Fontainebleau pour avoir exécuté des figures de stuc. On constate donc à cette occasion que, dans le domaine de la sculpture, les chantiers dirigés par les deux grands architectes de Henri II n'étaient pas étanches. On a cherché à restituer les carrières respectives de Jean et Antoine Chrétien, en étudiant avec les auditeurs un certain nombre d'actes inédits les concernant et en préparant les notices qui leur seront consacrées dans le Dictionnaire de l'art parisien de la Renaissance.

Un autre sculpteur présent sur le chantier d'Anet est Luc Jacquart. Sa participation au décor de la chapelle funéraire de Diane de Poitiers, en collaboration avec Mathieu Jacquet, avait été examinée dans les conférences d'histoire de Paris il y a quelques années. On avait alors évoqué la possibilité d'une intervention plus ancienne de l'artiste, dont on ne trouve pas trace à Paris avant 1564, et qui n'y fut reçu maître qu'en 1566. Un document nouveau vient aujourd'hui renforcer cette hypothèse : parmi les témoins attestant en 1570 bien connaître Robert Heisse et Laurent Lucas, respectivement peintre et sculpteur à Anet, figure Luc Jacquart qui affirme les avoir vu travailler depuis vingt ans pour le roi, ce qui correspond à la période de pleine activité du chantier dirigé par Philibert Delorme. Là encore, on s'est efforcé de retracer le parcours professionnel de l'artiste, documenté à partir de 1554, date à laquelle Pierre Bontemps lui sous-traite la taille de l'un des priants du tombeau de François I ${ }^{\text {er }}$. Ce n'est ensuite 
qu'à partir de 1564 qu'il est régulièrement mentionné dans les archives parisiennes, et pendant la décennie précédente il pourrait avoir travaillé régulièrement à Anet. Les commandes qu'on lui connaît par la suite, notamment sa participation à l'exécution des collèges apostoliques de la chapelle des Orfèvres et de l'église Saint-Barthélemy, montre qu'il était tout à fait capable d'exécuter de grandes statues comme les apôtres de la chapelle de Philibert Delorme.

D'autres séances ont été consacrées à l'établissement de notices biographiques d'artistes. Pour rédiger celle de Germain Pilon, on a été amené à remettre en cause la chronologie traditionnellement admise depuis la publication en 1951 par Madeleine Connat et Pierre du Colombier, dans la Bibliothèque d'Humanisme et Renaissance, d'un acte notarié daté du 16 septembre 1540, par lequel André Pilon faisait un don à son fils escollier, étudiant en l'Université de Paris. Pour les éditeurs de ce document, Germain, désigné comme écolier, ne pouvait avoir moins de 12 ans à cette date, ce qui situerait sa date de naissance avant 1528. Cette interprétation se trouvait en contradiction avec un autre texte, connu depuis longtemps, par lequel l'artiste, appelé comme témoin devant la Cour des monnaies, se déclarait en 1581 âgé de 46 ans ou environ. Du Colombier trancha la question de façon un peu rapide en prétendant « qu'on ne saurait ajouter qu'une foi très médiocre aux indications données comme approximatives par quelque greffier inattentif qui enregistre la déclaration d'un témoin qui a peut-être intérêt à se rajeunir ou à se vieillir ». L'argument fut repris par Ciprut pour qui Pilon, né en 1525, « avait dû chercher à se rajeunir ». Mais dans quel but aurait-il fait, sous serment, une fausse déclaration qui risquait de discréditer l'ensemble de son témoignage en faveur d'Alexandre Olivier, fils de son ami Aubin Olivier? La coquetterie est d'autant moins probable que le sculpteur avait pour sa part fait l'objet d'une enquête lorsqu'il était entré à la Monnaie, et que son âge ne pouvait être ignoré par la Cour.

Reste l'erreur possible du scribe invoquée par Du Colombier. On ne peut évidemment l'exclure, mais les minutes de la Cour des monnaies ne sont pas, comme il le prétend, des plumitifs où les textes sont saisis à la hâte : elles sont au contraire rédigées d'une écriture posée qui rend peu vraisemblable le lapsus calami, ou du moins qui requiert, pour l'envisager, de solides arguments. Or, l'acte de 1540 n'en constitue pas un. En effet, il ne signifie pas nécessairement que Germain Pilon ait eu plus de 12 ans à cette date : les statuts de l'Université réformés sous le règne de Henri IV défendirent de recruter à l'avenir des étudiants qui n'aient pas neuf ans révolus, ce qui laisse entendre que la pratique existait auparavant. Et, de fait, les minutes notariales mentionnent fréquemment des donations faites en faveur d'écoliers de moins de dix ans, surtout dans le monde des métiers : on le constate lorsque, quelques années plus tard, on les retrouve comme apprentis. La carrière de Germain Pilon s'accorde d'ailleurs mieux avec une date de naissance proche de 1534 ou 1535 que dix ans plus tôt. En effet, il était déjà maître en 1557, mais il l'était sans doute depuis peu car il travaillait encore à cette date avec son père pour des sculptures commandées par le président de Saint-André, alors que dans les années suivantes, chacun reçut des commandes séparées. Deux ans plus tard, naissait son premier fils, Raphaël. En 1573, l'orfèvre Richard Toutain, qui fournit une attestation en sa faveur, indiquait qu'il avait commencé dixhuit ans auparavant à fournir des modèles aux orfèvres, ce qui, là encore, situe le début de son activité professionnelle vers 1555 . C'est donc selon toute vraisemblance un 
jeune homme qui n'avait pas encore vingt-cinq ans qui commença à travailler pour le roi en 1559. Jusqu'en 1565, il habita la maison voisine de celle de son père au faubourg Saint-Jacques et il est probable que leur collaboration ne s'est pas brusquement arrêtée. Ainsi pourrait s'expliquer la ressemblance entre la tête provenant du gisant de Jean d'Estouteville, commandé à André Pilon en 1564, et celle du gisant de Henri II à Saint-Denis.

Un problème d'état civil similaire, mais plus aisé à résoudre, se rencontre avec la biographie de Jacques Androuet du Cerceau. L'historiographie traditionnelle le fait naître vers 1520 (ou même en 1520) en s'appuyant sur le fait qu'il était majeur à la mort de son père en 1546. Or, la seule conclusion que l'on peut tirer de ce document, comme de tous les autres connus jusqu'ici, est qu'il avait au moins 25 ans en 1546. Mais il pouvait avoir plus, et il n'y a donc aucune raison de mettre en doute sa déclaration lorsqu'en avril 1584, apportant son témoignage en faveur d'un charpentier protestant emprisonné à la Conciergerie, il se dit âgé de 73 ans. Il est donc né en 1511, et avait déjà 28 ans en 1539 lorsqu'il grava, au dire de La Croix du Maine, une Description de tout le pays et comté du Maine. Cette correction est importante pour éclairer sa formation et ses premiers rapports avec les artistes de Fontainebleau, mais c'est surtout un élément qui doit être pris en compte pour examiner les eaux-fortes et dessins de la « première manière » de Du Cerceau, dont certains sont datés du début des années 1530 , et qui pour cette raison lui ont parfois été retirés.

\section{Le théâtre à Paris au XVI siècle}

On a poursuivi cette année l'étude des mystères joués à Paris sous le règne de François I ${ }^{\mathrm{er}}$, en examinant les représentations des Actes des Apôtres de 1541 et du Viel Testament l'année suivante. Elles marquèrent les esprits : en 1549, pour situer un fait qui s'était déroulé sept ans plus tôt, un témoin ne donne pas le millésime mais mentionne l'année que l'on joua les jeux du Viel Testament. Plusieurs documents inédits venant compléter ceux déjà publiés et utilisés par Graham Runnalls, ont été présentés aux auditeurs, des contrats montrant qu'en 1542 plusieurs rôles furent tenus par des comédiens rémunérés. Ceux-ci n'étaient pas pour autant professionnels : Richard Georges, sergent au bailliage du Palais, et Thomas de Caniveau, compagnon ceinturier, touchèrent respectivement 40 et 35 écus pour « protocoler » le texte et diriger le jeu, mais Georges devait aussi jouer le personnage de Dieu dans les deux premières journées, puis ceux d'Abraham et de Cordelamor. Canivet s'engagea à tenir le rôle de Caïn, puis un autre qui n'était pas encore fixé à la signature du contrat.

On s'est attaché à examiner la personnalité des organisateurs de ces deux mystères. À cette date, ils ne portent plus, provisoirement, le titre de confrères de la Passion sans doute à la suite de l'interdiction des confréries professionnelles par l'édit de VillersCotterêts en 1539, mais ils figurent bien dans la liste des membres lorsque la confrérie réapparut officiellement au début du règne de Henri II. En 1542, ils sont désignés comme maîtres et entrepreneurs du jeu et mystère du Viel Testament : ce sont Isaac Cholet, marchand, François Pourtrain, marchand tapissier, Pierre Hemon, huissier à la Cour des aides, Charles Le Royer, marchand de poisson de mer et Gratien Berthier, maître pourpointier. L'inventaire de la femme de ce dernier, rédigé entre le 27 septembre 
et le 2 octobre 1542, alors que le jeu n'était pas encore fini, est instructif. On constate que la fortune mobilière du couple, 725 livres, dont un tiers de marchandises, était relativement faible par rapport à la somme que les quatre entrepreneurs avaient engagée dans l'opération : en décembre 1541, avant le début du jeu, et alors que le Parlement menaçait d'interdire les représentations, ils avaient déjà avancé 7000 livres à eux quatre. Le peintre Jean Cousin, à lui seul, devait recevoir 2000 livres pour les décors. D'autres confrères avaient dû contribuer : parmi les créanciers de Berthier, on relève les noms de Jean Fade et Adrien Gervais, deux des entrepreneurs de 1529.

On s'est également penché cette année sur la personnalité de Nicolas Hervé, dit Venise, maçon et fainteur parisien, appelé en 1541 à Châteauneuf-en-Gâtinais pour concevoir et exécuter les décors et les truquages de la Resurection, Assencion et Aparition du Saint Esperit aux apostres, Trespasement de Nostre Dame et Vengeance de la Passion. Il est, lui aussi, mentionné en 1548 parmi les confrères de la Passion. 